\title{
Cross Sections for Photoionization from Valence-Electron States
}

\author{
A. Burgess and M. J. Seaton \\ Department of Physics, University College, University of London, London, England
}

$\mathbf{I}^{\mathrm{N}}$ $\mathrm{N}$ order to study recombination processes in gaseous nebulae, one requires the coefficients for radiative recombination to many excited states of neutral atoms and positive ions. These may be calculated as functions of electron temperature once the corresponding photoionization cross sections are known. ${ }^{1}$ For photoionization from valence-electron states a general formula has been obtained using a method which is an extension of that used by Bates and Damgaard ${ }^{2}$ for bound-bound transition probabilities.

Rydberg energy units are used (13.60 ev or 109737 $\left.\mathrm{cm}^{-1}\right)$. Let $I_{n l}$ be the threshold energy for ejection of electron $n l ; k^{2}$ the kinetic energy of the ejected electron; the photon energy is then $\left(I_{n l}+k^{2}\right)$. The effective quantum number $n_{l}{ }^{*}$ is defined by $I_{n l}=Z^{2} / n_{l}{ }^{* 2}$ and the quantum defect by $\mu_{l}\left(-I_{n l}\right)=n_{l}{ }^{*}-n, Z$ being the residual charge on the ion. For the continuum states

TABLE I.

\begin{tabular}{ccccrcc}
\hline \hline$l$ & $l^{\prime}$ & \multicolumn{1}{c}{$a_{l l^{\prime}}$} & \multicolumn{1}{c}{$b_{l l^{\prime}}$} & \multicolumn{1}{c}{$c_{l l^{\prime}}$} & \multicolumn{1}{c}{$\alpha_{l l^{\prime}}$} & \multicolumn{1}{c}{$\boldsymbol{\beta}_{l l^{\prime}}$} \\
\hline $\mathbf{0}$ & 1 & -0.147 & +0.2515 & -0.078 & +0.310 & 0.000 \\
1 & 0 & -0.216 & -0.171 & 0.000 & 0.000 & 0.000 \\
1 & 2 & -0.120 & +0.600 & 0.000 & +0.362 & +0.0535 \\
2 & 1 & -0.247 & -0.272 & 0.000 & -0.010 & -0.019 \\
2 & 3 & -0.117 & +1.170 & 0.000 & +0.321 & +0.106 \\
3 & 2 & -0.362 & +0.599 & -2.432 & -0.390 & +0.050 \\
\hline \hline
\end{tabular}

TABLE II.

\begin{tabular}{rcccccc}
\hline \multicolumn{7}{c}{$\gamma_{n l^{\prime}\left(n l^{*}\right)}$} \\
$n \imath^{*}\left(l, l^{\prime}\right)=(0,1)$ & $(1,0)$ & $(1,2)^{(2,1)}$ & $(2,3)$ & $(3,2)$ \\
\hline 1 & 1.754 & $\ldots$ & $\ldots$ & $\ldots$ & $\ldots$ & $\ldots$ \\
2 & 1.605 & 1.667 & 1.574 & $\ldots$ & $\ldots$ & $\ldots$ \\
3 & 1.591 & 1.667 & 1.582 & 1.819 & 1.447 & $\ldots$ \\
4 & 1.590 & 1.667 & 1.579 & 1.771 & 1.535 & 1.850 \\
5 & 1.591 & 1.667 & 1.582 & 1.741 & 1.544 & 1.908 \\
6 & 1.594 & 1.667 & 1.587 & 1.722 & 1.549 & 1.918 \\
7 & 1.596 & 1.667 & 1.593 & 1.707 & 1.556 & 1.920 \\
8 & 1.599 & 1.667 & 1.598 & 1.697 & 1.564 & 1.921 \\
9 & 1.601 & 1.667 & 1.603 & 1.688 & 1.573 & 1.922 \\
10 & 1.603 & 1.667 & 1.608 & 1.682 & 1.581 & 1.924 \\
11 & 1.605 & 1.667 & 1.614 & 1.676 & 1.589 & 1.926 \\
12 & 1.607 & 1.667 & 1.618 & 1.672 & 1.596 & 1.928 \\
& & & & & & \\
\hline \hline
\end{tabular}

${ }^{1}$ M. J. Seaton, Monthly Notices Roy. Astron. Soc. 111, 368 (1951).

2 D. R. Bates and A. Damgaard, Phil. Trans. Roy. Soc. London A242, 101 (1949). put $k^{2}=Z^{2} / \theta^{2}$. The photoionization cross section is

$a_{v}(n l)=8.57 \times 10^{-19} \frac{\left(I_{n l}+k^{2}\right)}{I_{n l^{2}}} \sum_{l^{\prime}=l \pm 1} C_{l^{\prime}}\left|g\left(n_{l}^{*} ; \theta, l^{\prime}\right)\right|^{2} \mathrm{~cm}^{2}$,

where, for alkali-like systems, $C_{l-1}=l /(2 l+1)$ and $C_{l+1}=(l+1) /(2 l+1)$. The matrix element is

$$
g\left(n_{l}^{*} ; \theta, l^{\prime}\right)=I_{n l} \int_{0}^{\infty} P\left(n_{l}^{*} ; r\right) r F\left(k, l^{\prime}, Z ; r\right) d r
$$

$P\left(n_{l}{ }^{*} ; r\right)$ being the normalized bound-state radial function and $F\left(k, l^{\prime}, Z ; r\right)$ the continuum radial function having the asymptotic form

$$
\begin{aligned}
F\left(k, l^{\prime}, Z ; r\right) \sim k^{-\frac{1}{2}} \sin \left[k r-\frac{1}{2} l^{\prime} \pi+\frac{Z}{k} \ln (2 k r)\right. & \\
& \left.+\arg \Gamma\left(l^{\prime}+1-\frac{i Z}{k}\right)+\delta_{l^{\prime}}\left(k^{2}\right)\right] .
\end{aligned}
$$

The phase $\delta_{l^{\prime}}\left(k^{2}\right)$ is zero for the pure Coulomb field.

The general formula depends on the relation $\delta_{l^{\prime}}\left(k^{2}\right)$ $=\pi \mu_{l^{\prime}}\left(k^{2}\right)$, valid for $k \ll(2 \pi Z)$, where $\mu_{l^{\prime}}\left(k^{2}\right)$ is the quantum defect for the $n^{\prime} l^{\prime}$ series extrapolated as a function of energy., ${ }^{3,4}$ The Bates and Damgaard functions $P\left(n_{l}^{*} ; r\right)$ have been used together with approximate analytic functions $F\left(k, l^{\prime}, Z ; r\right)$ having

TABLE III.

\begin{tabular}{ccccccc}
\hline \multicolumn{7}{c}{$G_{n l^{\prime}\left(n l^{*}\right)}$} \\
$n^{*}\left(l, l^{\prime}\right)=(0,1)$ & $(1,0)$ & $(1,2)$ & $(2,1)$ & $(2,3)$ & $(3,2)$ \\
\hline 1 & 2.723 & $\ldots$ & $\ldots$ & $\ldots$ & $\ldots$ & $\ldots$ \\
2 & 2.095 & 1.028 & 2.840 & $\ldots$ & $\ldots$ & $\ldots$ \\
3 & 1.856 & 1.117 & 2.264 & 0.669 & 3.000 & $\ldots$ \\
4 & 1.718 & 1.152 & 2.010 & 0.818 & 2.413 & 0.468 \\
5 & 1.623 & 1.168 & 1.856 & 0.899 & 2.139 & 0.599 \\
6 & 1.553 & 1.175 & 1.749 & 0.952 & 1.971 & 0.704 \\
7 & 1.498 & 1.177 & 1.666 & 0.988 & 1.854 & 0.793 \\
8 & 1.452 & 1.176 & 1.601 & 1.014 & 1.765 & 0.868 \\
9 & 1.414 & 1.173 & 1.546 & 1.033 & 1.694 & 0.933 \\
10 & 1.381 & 1.170 & 1.501 & 1.047 & 1.635 & 0.991 \\
11 & 1.352 & 1.165 & 1.461 & 1.058 & 1.585 & 1.041 \\
12 & 1.327 & 1.161 & 1.427 & 1.065 & 1.543 & 1.085 \\
& & & & & & \\
\hline \hline
\end{tabular}

${ }^{3}$ M. J. Seaton, Compt. rend. 240, 1317 (1955); Proc. Phys. Soc. (London) A70, 620 (1957).

${ }^{4}$ F. S. Ham, Solid State Physics, edited by F. Seitz and D. Turnbull (Academic Press, Inc., New York, 1955), Vol. 1, p. 127. 
correct asymptotic form. Extensive numerical calculations have been made for $\left(l, l^{\prime}\right)=(0,1),(1,0),(1,2)$, $(2,1),(2,3)$, and $(3,2)$ and $(l+1) \leqslant n_{l}{ }^{*} \leqslant 12$. The results obtained may be fitted to expressions of the form

$$
\begin{aligned}
& g\left(n_{l}^{*} ; \theta, l^{\prime}\right)=G_{l l^{\prime}}\left(n_{l^{*}}^{*}\right)[1\left.+\left(n_{l^{*}}^{*} / \theta\right)^{2}\right]^{-\gamma l l^{\prime}\left(n l^{*}\right)} \\
& \times \cos \pi\left[n_{l}^{*}+\mu_{l^{\prime}}\left(k^{2}\right)+\chi_{l l^{\prime}}\left(n_{l^{*}}^{*}, \theta\right)\right], \\
& \chi_{l l^{\prime}}\left(n_{l^{*}}^{*}, \theta\right)=a_{l l^{\prime}}+\frac{b_{l l^{\prime}}}{n_{l^{*}}}+\frac{c_{l l^{\prime}}}{n_{l}{ }^{* 2}}+\frac{\left(n^{*} / \theta^{2}\right) \alpha_{l l^{\prime}}}{1+\left(n_{l}^{*} / \theta^{2}\right)}+\frac{\left(n^{*} / \theta\right)^{2} \beta_{l l^{\prime}}}{1+\left(n_{l}^{*} / \theta\right)^{2}} .
\end{aligned}
$$

In the approximation used $g$ depends on $\theta^{2}$ and on the effective quantum numbers but is otherwise independent of $Z$. The coefficients $a_{l l^{\prime}}, b_{l l^{\prime}}, c_{l l^{\prime}}, \alpha_{l l^{\prime}}$ and $\beta_{l l^{\prime}}$ are given in Table I. The functions $G_{l l^{\prime}}\left(n_{l}{ }^{*}\right)$ and $\gamma_{l l^{\prime}}\left(n_{l}{ }^{*}\right)$ vary slowly with $n_{l}{ }^{*}$ and may be interpolated from the values for integer $n_{l}^{*}$ given in Tables II and III.

The bound-bound matrix elements

$$
\rho\left(n_{l}{ }^{*}, n_{l}{ }^{*}\right)=\int P\left(n_{l}{ }^{*} ; r\right) r P\left(n_{l}{ }^{*} ; r\right) d r
$$

may be calculated for $n_{l} * \gg n_{l} *$ using the relation

$$
\rho\left(n_{l}{ }^{*}, n_{l}{ }^{*}\right)=\left(n_{l}^{* 2} / Z n_{l^{\prime}}{ }^{* \frac{3}{3}}\right)(2 / \pi)^{\frac{1}{2}} g\left(n_{l}{ }^{*} ; i n_{l^{\prime}}{ }^{*}, l^{\prime}\right) .
$$

For $n_{l} * \geq\left(n_{l}^{*}+1.5\right)$ this gives results not differing significantly from those obtained by Bates and Damgaard. The accuracy of the photoionization general formula should be comparable with that of the Bates and Damgaard calculations for bound-bound transition probabilities. 